\title{
Anatomia quantitativa do atlas
}

\author{
Max Franco de Carvalho', Roberta Teixeira Rocha², João Tiago Silva Monteiro ${ }^{3}$, Carlos \\ Umberto Pereira ${ }^{4}$, Alex Franco de Carvalho ${ }^{5}$, Helton Luiz Aparecido Defino 6
}

Faculdade de Medicina da Universidade Federal de Sergipe e Faculdade de Medicina de Ribeirão Preto da Universidade de São Paulo, Brasil

Este trabalho foi realizado com auxílio de Bolsas de Iniciação Científica Pibic/Capes/CNPq

\section{RESUMO}

Objetivo: Descrever as medidas anatômicas gerais do atlas por meio do estudo anatômico e por tomografia computadorizada helicoidal em 30 amostras do atlas isolado de cadáveres adultos brasileiros. Métodos: Todos os parâmetros observados foram analisados estatisticamente para a obtenção da média, desvio-padrão, o valor mínimo e o máximo das estruturas do atlas. Resultados: A reprodutibilidade foi considerada boa para coeficiente de correlação $>0,80$ e ruim para coeficiente $<0,80$. Em 12 parâmetros, a reprodutibilidade foi considerada boa. Apenas nos parâmetros da distância interligamento transverso e na dimensão do espaço odontóide, a reprodutibilidade foi considerada ruim, apresentando coeficientes de correlação interclasse < 0,06. Conclusão: Os dados obtidos fornecem um melhor entendimento de geometria, dimensões e detalhes quantitativos de morfometria atlantal. Este estudo fornece as dimensões gerais do atlas e demonstra uma proporção constante dos arcos anterior e posterior. Os autores acreditam que essas observações possam facilitar os estudos biomecânicos, radiológicos e cirúrgicos da transição craniocervical.

\section{PALAVRAS-CHAVE}

Atlas. Anatomia.

\section{ABSTRACT}

\section{Quantitative anatomy of the athas}

Objective: To describe the general anatomical measures of atlas through the anatomical observation and by helicoidal computed tomography scanning of 30 samples of isolated Brazilian adult atlas. Methods: All the observed parameters were statistically analyzed. Results: The reproducibility was considered good for correlation coefficient $>0.80$ which were achieved in twelve parameters measured. This was not true for measurements obtained for the intertransverse ligament distance and for the dimension of the odontoidal space. Conclusion: The data obtained will provide a better knowledge of the geometry dimensions of the atlas morphometry. The authors believe that those data may be contribute to the biomechanical and radiological studies as to the surgery of the craniocervical transition.

\section{KEY WORDS}

Atlas. Anatomy.

\section{Introdução}

A transição craniocervical é a região da coluna cervical responsável pelo segmento de maior mobilidade da coluna vertebral. Devido a essa importância funcional única, a primeira vértebra cervical - o atlas - apresenta características morfológicas diferentes das demais vértebras da coluna e é considerada atípica ${ }^{1,3,7-9,11,16,17}$. A principal característica do atlas é a ausência do corpo vertebral e do processo espinhoso. Essa vértebra apresenta estrutura em forma de anel, formada por duas massas laterais conectadas por um arco anterior curto e outro longo posterior.

\footnotetext{
1 Aluno do Programa de Pós-Graduação (Doutorado) da Faculdade de Medicina de Ribeirão Preto da Universidade de São Paulo. 2 Médica do Setor de Radiologia da Universidade Federal de Sergipe.

3 Doutorando de Medicina da Universidade Federal de Sergipe. Aluno bolsista de Iniciação Científica Pibic/Capes/CNPq. 4 Professor doutor adjunto do Departamento de Medicina da Universidade Federal de Sergipe. 5 Professor doutor adjunto do Departamento de Medicina da Universidade Federal de Sergipe. 6 Professor doutor associado do Departamento de Biomecânica, Medicina e Reabilitação do Aparelho Locomotor da Faculdade de Medicina de Ribeirão Preto, da Universidade de São Paulo.
} 
Com o desenvolvimento do diagnóstico e a precisão das técnicas cirúrgicas de fusão da articulação atlantoaxial, existem diversos estudos biomecânicos e clínico-cirúrgicos dessa região, enfatizando principalmente a estabilidade conseguida por diferentes técnicas de instrumentação $0^{2,5,6,10,12,14,15,18,20,22}$. Entretanto, existem poucos relatos na literatura enfatizando aspectos dimensionais, importância morfofuncional e cirúrgica do atlas.

O objetivo deste trabalho é descrever a anatomia detalhada de 30 atlas ressecados, de origem brasileira, por meio de observações anatômicas e radiológicas, com o uso de tomografia computadorizada helicoidal. As avaliações desses resultados poderão servir como base para estudos biomecânicos, desenvolvimento de materiais de osteossíntese e facilitação da abordagem cirúrgica dessa região.

\section{Material e métodos}

Trinta vértebras cervicais ( $\mathrm{C} 1$ ou atlas) de cadáveres adultos não-identificados foram obtidas por meio de doação da Empresa Municipal de Serviços Urbanos (EMURB) de Aracaju, Sergipe, Brasil, pelo parecer $\mathrm{n}^{\circ} 047 / 2005$. As vértebras eram provenientes de indivíduos de origem local, sepultados como indigentes, e não houve distinção quanto a sexo, idade ou raça. Após dissecação, limpeza e fixação, as vértebras foram numeradas para posterior análise de medidas anatômicas manuais com paquímetro digital quadridimensional (Starret SR44, com precisão de $0,01 \mathrm{~mm}$ ) e radiológicas, por tomografia computadorizada helicoidal (Toshiba Asteion TSX - 021A/1A, cortes com espessura de $1 \mathrm{~mm}$ ).

Os parâmetros anatômicos medidos por tomografia e estudo anatômico foram: altura do arco anterior (Aa), espessura do arco anterior (Ea), altura do arco posterior (Ap) e espessura do arco posterior (Ep) (figura 1); diâmetro transverso interno (DTi), diâmetro longitudinal interno (DLi), diâmetro transverso externo (DTe), diâmetro longitudinal externo (Dle), diâmetro longitudinal do forâmen transverso (DLf), diâmetro transverso do forâmen transverso (DTf), distância interligamento transverso (Dil) e espaço odontóide (Eo) (figura 2).

Para avaliar a reprodutibilidade das mensurações realizadas, as medidas anatômicas foram realizadas por dois observadores, sem conhecimento dos resultados do primeiro observador. Foi selecionado aleatoriamente um subgrupo de cinco vértebras, que foram submetidas a novo exame tomográfico, no qual as medidas foram realizadas uma segunda vez pelo mesmo observador, sem conhecimento dos resultados da primeira mensuração. Para a análise da variação interobservador e intraobservador e com o estudo radiológico, foi calculado o coeficiente de correlação intraclasses $(\mathrm{CCI})^{19,21}$.
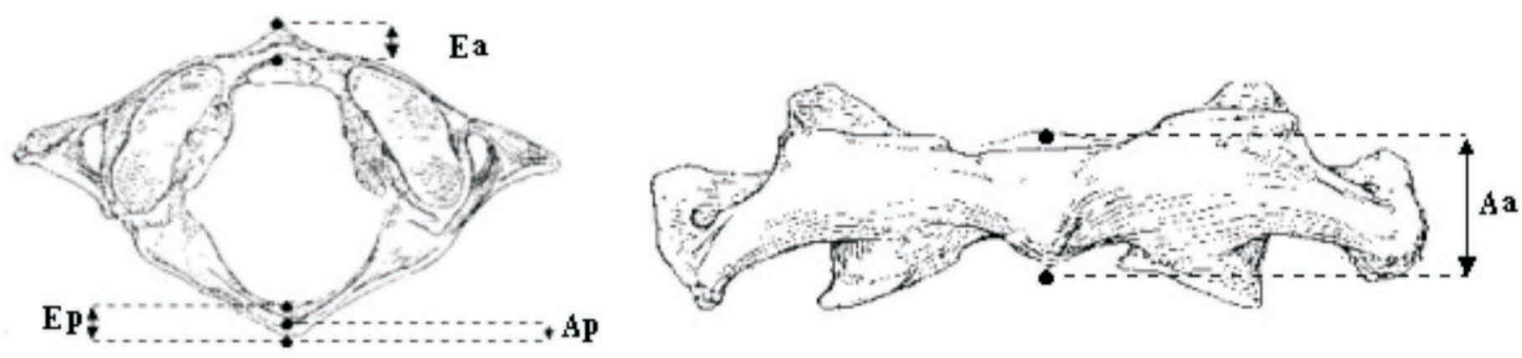

Figura 1 - Estudos dos arcos anteriores e posteriores do atlas.
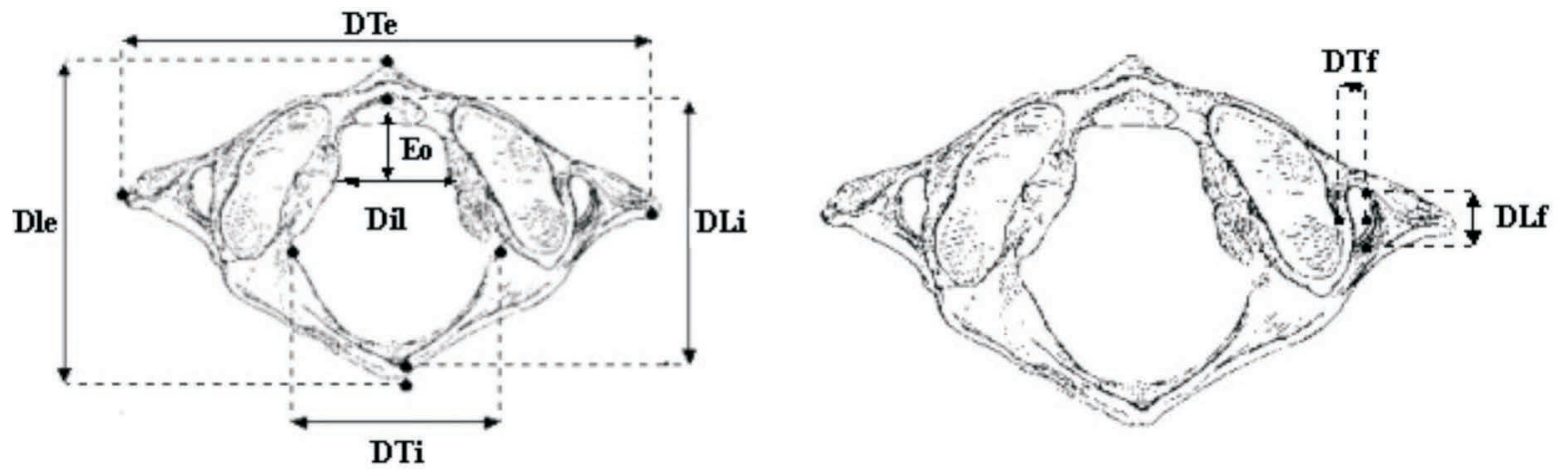

Figura 2 - Estudo dos diâmetros do atlas. 
A consistência entre as medidas realizadas pelos dois observadores e pela radiologia foi avaliada pelo $\mathrm{CCI}^{19,21}$ e respectivo intervalo de confiança (95\%). Para a comparação das variáveis avaliadas dos lados direito e esquerdo, foi adotado o modelo de regressão linear. Nos casos em que mais de uma medida era realizada em uma mesma vértebra por métodos (ou pesquisadores) diferentes (observadores 1 e 2 e radiologista), o modelo possibilitou o ajuste de uma reta para cada método. A igualdade dos lados foi avaliada pelo teste da hipótese de que o intercepto da reta era igual a 0 e de que o coeficiente angular era igual a 1 .

\section{Resultados}

Com base nos achados das medidas dos 30 atlas, foram calculados mediana, média, valor máximo, valor mínimo, desvio-padrão e o coeficiente de correlação intraclasse.

As médias observadas para a altura do arco anterior foram de 10,66 $\pm 1,03 \mathrm{~mm}$ pelos observadores e de 10,23 $\pm 1,13 \mathrm{~mm}$ na radiologia. As médias observadas da espessura do arco anterior foram de $6,35 \pm 0,79 \mathrm{~mm}$ pelos observadores e 5,96 $\pm 0,96 \mathrm{~mm}$ na radiologia (tabela $1 \mathrm{~A}$ ). Para a altura do arco posterior, as médias obtidas foram de 8,48 $\pm 1,37 \mathrm{~mm}$ pelos observadores e $8,66 \pm 1,49 \mathrm{~mm}$ na radiologia. As médias obtidas da espessura do arco posterior foram de $6,16 \pm 2,29 \mathrm{~mm}$ pelos observadores e 5,36 $\pm 1,79 \mathrm{~mm}$ na radiologia (tabela $1 \mathrm{~B}$ ).

As medidas médias do diâmetro longitudinal interno foram de $31,11 \pm 2,26 \mathrm{~mm}$ obtidas pelos observadores e de $30,65 \pm 2,13 \mathrm{~mm}$ na radiologia. Para a medida do diâmetro transverso interno, a média observada pelos observadores foi de 28,45 $\pm 2,31 \mathrm{~mm}$ e $31,12 \pm 3,04 \mathrm{~mm}$ na radiologia (tabela $2 \mathrm{~A})$. As médias do diâmetro longitudinal externo foram de 44,07 $\pm 2,48 \mathrm{~mm}$ determinadas pelos observadores, e 42,34 $\pm 2,63 \mathrm{~mm}$ na radiologia. Para a medida do diâmetro transverso externo, a média obtida pelos observadores foi de 75,89 $\pm 4,76 \mathrm{~mm}$ e $75,20 \pm 4,71 \mathrm{~mm}$ pela radiologia (tabela $2 \mathrm{~B}$ ).

As medidas obtidas para os diâmetros longitudinal e transverso do forâmen transverso direito e esquerdo não apresentaram discrepâncias significativas tanto no estudo anatômico como no estudo radiológico. A média observada pelos observadores do diâmetro transverso do forâmen transverso foi de $6,45 \pm 1,12 \mathrm{~mm}$ à direita e $6,68 \pm 1,30 \mathrm{~mm}$ na radiologia. As médias observadas à esquerda foram de $6,48 \pm 1,17 \mathrm{~mm}$ e $6,93 \pm 1,37 \mathrm{~mm}$, respectivamente (tabela $3 \mathrm{~A}$ ). Para o diâmetro longitudinal do forâmen transverso, a média observada à direita pelos observadores foi $6,86 \pm 1,27 \mathrm{~mm}$ e 7,72 $\pm 1,28 \mathrm{~mm}$ na radiologia e à esquerda foi de $6,73 \pm 1,20 \mathrm{~mm}$ e 7,55 $\pm 1,23 \mathrm{~mm}$, respectivamente (tabela $3 \mathrm{~B}$ ).

\begin{tabular}{|c|c|c|c|c|c|c|c|c|}
\hline \multicolumn{9}{|c|}{$\begin{array}{l}\text { Tabela } 1 \mathrm{~A} \\
\text { Espessura e altura do arco anterior em milímetros }\end{array}$} \\
\hline & \multicolumn{4}{|c|}{ Espessura arco anterior } & \multicolumn{4}{|c|}{ Altura arco anterior } \\
\hline & \multicolumn{4}{|c|}{ Obs 1 Obs 2 Média Radiologia } & Obs 1 & \multicolumn{2}{|c|}{ Obs 2 Média } & Radiologia \\
\hline Mínimo & 4,81 & 4,91 & 4,88 & 4,00 & 8,61 & 8,56 & 8,59 & 8,00 \\
\hline Máximo & 8,24 & 8,29 & 8,26 & 8,10 & 12,56 & 12,46 & 12,51 & 12,70 \\
\hline Mediana & 6,25 & 6,27 & 6,26 & 5,85 & 10,85 & 10,84 & 10,85 & 10,25 \\
\hline Média & 6,34 & 6,36 & 6,35 & 5,96 & 10,66 & 10,66 & 10,66 & 10,23 \\
\hline DP & 0,80 & 0,79 & 0,79 & 0,96 & 1,02 & 1,03 & 1,03 & 1,13 \\
\hline $\mathrm{CCI}$ & & & 0,87 & & & & 0,93 & \\
\hline IC $95 \%$ & & & $, 76 ; 0,93]$ & & & &, $88 ; 0,97$ & \\
\hline $\mathrm{p}$ & & & $<0,0001$ & & & & $<0,0001$ & \\
\hline \multicolumn{9}{|c|}{$\begin{array}{l}\text { CCI: Coeficiente de correlação interclasses; IC: Índice de confiabilidade; } \\
\text { Obs.: observador; DP: Desvio-padrão. }\end{array}$} \\
\hline \multicolumn{9}{|c|}{$\begin{array}{c}\text { Tabela } 1 \mathrm{~B} \\
\text { Espessura e altura do arco posterior em milímetros }\end{array}$} \\
\hline \multicolumn{5}{|c|}{ Espessura arco posterior } & \multicolumn{4}{|c|}{ Altura arco posterior } \\
\hline & Obs 1 & Obs 2 & Média $\mathbf{R}$ & Radiologia & Obs 1 & Obs 2 & Média & Radiologia \\
\hline Mínimo & 3,40 & 3,42 & 3,41 & 2,10 & 6,47 & 6,52 & 6,50 & 6,70 \\
\hline Máximo & 11,95 & 11,93 & 11,94 & 9,60 & 14,16 & 14,12 & 14,14 & 13,70 \\
\hline Mediana & 5,43 & 5,38 & 5,41 & 5,10 & 8,31 & 8,29 & 8,30 & 8,50 \\
\hline Média & 6,16 & 6,15 & 6,16 & 5,36 & 8,48 & 8,48 & 8,48 & 8,66 \\
\hline DP & 2,29 & 2,29 & 2,29 & 1,79 & 1,38 & 1,37 & 1,37 & 1,49 \\
\hline CCI & & & 0,94 & & & & 0,97 & \\
\hline IC $95 \%$ & & & $, 88 ; 0,97]$ & & & &, $95 ; 0,95$ & \\
\hline $\mathrm{p}$ & & & $<0,0001$ & & & & $<0,0001$ & \\
\hline
\end{tabular}

CCI: Coeficiente de correlação interclasses; IC: Índice de confiabilidade; Obs.: observador; DP: Desvio-padrão.

\begin{tabular}{|c|c|c|c|c|c|c|c|c|}
\hline \multicolumn{9}{|c|}{$\begin{array}{c}\text { Tabela } 2 A \\
\text { Diâmetros internos do atlas em milímetros }\end{array}$} \\
\hline & \multicolumn{4}{|c|}{ Transverso interno } & \multicolumn{4}{|c|}{ Longitudinal interno } \\
\hline & Obs 1 & Obs 2 & Média & Radiologia & Obs 1 & Obs 2 & Média & Radiologia \\
\hline Mínimo & 23,91 & 23,89 & 23,90 & 26,70 & 27,31 & 27,33 & 27,32 & 27,10 \\
\hline Máximo & 33,63 & 33,61 & 33,62 & 37,00 & 36,07 & 36,07 & 36,06 & 34,60 \\
\hline Mediana & 28,35 & 28,34 & 28,35 & 31,10 & 30,56 & 30,55 & 30,56 & 30,25 \\
\hline Média & 28,44 & 28,44 & 28,45 & 31,12 & 31,11 & 31,11 & 31,11 & 30,65 \\
\hline DP & 2,31 & 2,32 & 2,31 & 3,04 & 2,26 & 2,26 & 2,26 & 2,13 \\
\hline $\mathrm{CCI}$ & & & 0,90 & & & & 0,98 & \\
\hline IC $95 \%$ & & & $82 ; 0,95$ & & & &, $97 ; 0,99$ & \\
\hline $\mathrm{p}$ & & & $<0,0001$ & & & & $<0,0001$ & \\
\hline
\end{tabular}

CCI: Coeficiente de correlação interclasses; IC: Índice de confiabilidade; Obs.: observador; DP: Desvio-padrão.

\begin{tabular}{|c|c|c|c|c|c|c|c|c|}
\hline \multicolumn{9}{|c|}{$\begin{array}{l}\text { Tabela } 2 B \\
\text { ernos do atlas em milímetros }\end{array}$} \\
\hline & \multicolumn{4}{|c|}{ Transverso externo } & \multicolumn{4}{|c|}{ Longitudinal externo } \\
\hline & Obs 1 & Obs 2 & Média & Radiologia & Obs 1 & 1 Obs 2 & Média & Radiologia \\
\hline Mínimo & 64,78 & 64,74 & 64,76 & 64,40 & 40,67 & 40,73 & 40,70 & 37,60 \\
\hline Máximo & 84,33 & 84,55 & 84,43 & 83,90 & 49,00 & 49,15 & 49,08 & 48,70 \\
\hline Mediana & 75,48 & 75,48 & 75,49 & 74,90 & 43,60 & 43,46 & 43,53 & 42,30 \\
\hline Média & 75,87 & 75,90 & 75,89 & 75,20 & 44,07 & 44,06 & 44,07 & 42,34 \\
\hline DP & 4,75 & 4,77 & 4,76 & 4,71 & 2,49 & 2,48 & 2,48 & 2,63 \\
\hline $\mathrm{CCI}$ & & & 0,99 & & & & 0,94 & \\
\hline IC $95 \%$ & & &, $97 ; 0,99$ & & & & 0,$9 ; 0,97$ & \\
\hline $\mathrm{p}$ & & & $<0,0001$ & & & & $<0,0001$ & \\
\hline
\end{tabular}

CCI: Coeficiente de correlação interclasses; IC: Índice de confiabilidade; Obs.: observador; DP: Desvio-padrão. 
Tabela 3 A

Diâmetros transversos do forâmen transverso em milímetros

Diâmetro transverso direito Diâmetro transverso esquerdo

Obs 1 Obs 2 Média Radiologia Obs 1 Obs 2 Média Radiologia

\begin{tabular}{lllllllll}
\hline Mínimo & 4,01 & 4,12 & 4,07 & 3,40 & 4,08 & 4,05 & 4,07 & 4,20
\end{tabular}

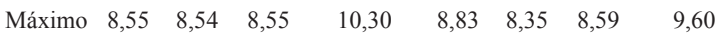

$\begin{array}{lllllllll}\text { Mediana } & 6,45 & 6,45 & 6,42 & 6,60 & 6,58 & 6,62 & 6,61 & 7,15\end{array}$

$\begin{array}{lllllllll}\text { Média } & 6,44 & 6,46 & 6,45 & 6,68 & 6,49 & 6,46 & 6,48 & 6,93\end{array}$

$\begin{array}{lllllllll}\text { DP } & 1,12 & 1,12 & 1,12 & 1,30 & 1,21 & 1,15 & 1,17 & 1,37\end{array}$

CCI $0,85 \quad 0,84$

IC $95 \% \quad[0,72 ; 0,92] \quad[0,71 ; 0,92]$

p $<0,0001<0,0001$

CCI: Coeficiente de correlação interclasses; IC: Índice de confiabilidade; Obs.: observador; DP: Desvio-padrão.

Tabela $3 B$

Diâmetros longitudinais do forâmen transverso em milímetros Diâmetro longitudinal direito Diâmetro longitudinal esquerdo Obs 1 Obs 2 Média Radiologia Obs 1 Obs 2 Média Radiologia

\begin{tabular}{lllllllll}
\hline Mínimo & 4,23 & 4,36 & 4,30 & 5,00 & 4,96 & 4,96 & 4,96 & 5,80
\end{tabular}

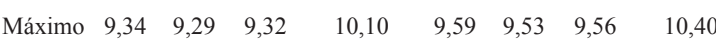

$\begin{array}{lllllllll}\text { Mediana } & 6,85 & 6,76 & 6,75 & 7,70 & 6,40 & 6,38 & 6,38 & 7,15\end{array}$

$\begin{array}{lllllllll}\text { Média } & 6,90 & 6,82 & 6,86 & 7,72 & 6,74 & 6,72 & 6,73 & 7,55\end{array}$

$\begin{array}{lllllllll}\text { DP } & 1,25 & 1,31 & 1,27 & 1,28 & 1,23 & 1,19 & 1,20 & 1,23\end{array}$

CCI $0,99 \quad 1,00$

IC $95 \% \quad[0,99 ; 1] \quad[0,99 ; 1]$

p $<0,0001<0,0001$

CCI: Coeficiente de correlação interclasses; IC: Índice de confiabilidade; Obs.: observador; DP: Desvio-padrão.

A média observada da distância interligamento transverso foi de $17,43 \pm 1,56 \mathrm{~mm}$ pelos observadores e 18,92 $\pm 2,97 \mathrm{~mm}$ na radiologia. Para a dimensão espaço do odontóide, as médias observadas foram de $8,86 \pm 0,86 \mathrm{~mm}$ e $7,93 \pm 1,14 \mathrm{~mm}$, respectivamente (tabela 4).

\begin{tabular}{|c|c|c|c|c|c|c|c|c|}
\hline \multicolumn{9}{|c|}{$\begin{array}{c}\text { Tabela } 4 \\
\text { Dimensões no espaço para o processo odontóide em milímetros }\end{array}$} \\
\hline & \multicolumn{4}{|c|}{$\begin{array}{c}\text { Distância interligamento } \\
\text { transverso }\end{array}$} & \multicolumn{4}{|c|}{ Dimensão espaço do odontóide } \\
\hline & Obs 1 & Obs 2 & Média & Radiologia & Obs 1 & Obs 2 & Média & Radiologia \\
\hline Mínimo & 14,98 & 14,93 & 14,96 & 14,60 & 7,53 & 7,48 & 7,51 & 6,50 \\
\hline Máximo & 21,01 & 21,65 & 21,33 & 26,20 & 11,23 & 11,67 & 11,45 & 10,80 \\
\hline Mediana & 17,65 & 17,55 & 17,60 & 18,50 & 8,81 & 8,79 & 8,69 & 7,60 \\
\hline Média & 17,40 & 17,46 & 17,43 & 18,92 & 8,83 & 8,88 & 8,86 & 7,93 \\
\hline DP & 1,52 & 1,61 & 1,56 & 2,97 & 0,84 & 0,90 & 0,86 & 1,14 \\
\hline $\mathrm{CCI}$ & \multicolumn{4}{|c|}{0,5758} & \multicolumn{4}{|c|}{0,51} \\
\hline IC $95 \%$ & \multicolumn{4}{|c|}{$[0,22 ; 0,78]$} & \multicolumn{4}{|c|}{$[0,1 ; 0,75]$} \\
\hline $\mathrm{p}$ & \multicolumn{4}{|c|}{0,0027} & \multicolumn{4}{|c|}{0,0102} \\
\hline
\end{tabular}

CCI: Coeficiente de correlação interclasses; IC: Índice de confiabilidade; Obs.: observador; DP: Desvio-padrão.

A reprodutibilidade foi considerada boa para coeficiente de correlação $>0,80$ e ruim para coeficiente $<0,80$. Em 12 parâmetros, a reprodutibilidade foi consi- derada boa. Apenas nos parâmetros da distância interligamento transverso e na dimensão do espaço odontóide, a reprodutibilidade foi considerada ruim, apresentando coeficientes de correlação interclasse $<0,06$.

\section{Discussão}

O número de procedimentos na articulação atlantoaxial tem aumentado bastante com o desenvolvimento dos meios diagnósticos, melhor entendimento da biomecânica e desenvolvimento dos métodos de fixação ${ }^{11,12}$. Os métodos de fixação variam desde amarrilha, fixação com parafuso transarticular, uso de ganchos, inserção de parafusos na massa articular ou translaminar no atlas $^{2,3,5,6,10,12-15,18,20,22}$. A lesão iatrogênica da artéria vertebral e a inserção de parafusos no canal medular são as complicações transoperatórias mais citadas ${ }^{15,18}$.

A literatura recente tem dado ênfase na descrição biomecânica e cirúrgica da coluna cervical alta. Entretanto, existem poucos artigos que descrevem a anatomia dos diâmetros do atlas. Doherty e Heggness ${ }^{4}$, em 1994, descreveram as dimensões de 80 vértebras de origem européia e observaram no diâmetro longitudinal e transverso externo médias de 45,8 $\pm 2,9 \mathrm{~mm}$ e 78,6 $\pm 8,1 \mathrm{~mm}$, respectivamente. Para os diâmetros longitudinal e transverso interno, observaram médias de $31,7 \pm 2,2 \mathrm{~mm}$ e 32,32 $\pm 2,3 \mathrm{~mm}$, respectivamente. Em nosso estudo, os diâmetros longitudinal e transverso externo foram de 44,07 $\pm 2,48 \mathrm{~mm}$ e 75,89 \pm 4,76 $\mathrm{mm}$ na média dos observadores e 42,34 $\pm 2,63 \mathrm{~mm}$ e 75,20 $\pm 4,71 \mathrm{~mm}$ na radiologia, respectivamente. Para a média observada dos diâmetros longitudinal e transverso internos, tivemos $31,11 \pm 2,26 \mathrm{~mm}$ e $28,45 \pm 2,31 \mathrm{~mm}$ da média dos observadores e $30,65 \pm 2,13 \mathrm{~mm}$ e 31,12 $\pm 3,04 \mathrm{~mm}$ na radiologia, respectivamente. Tanto as dimensões externas quanto as internas demonstraram diferenças menores do que um desvio-padrão, sugerindo uma equivalência dimensional nas vértebras brasileiras e européias.

$\mathrm{O}$ arco anterior demonstrou ser mais espesso e mais alto do que o posterior, possivelmente devido à carga biomecânica da articulação do atlas com o processo odontóide. Doherty e Heggness ${ }^{4}$ observaram histologicamente que a cortical do arco anterior era mais espessa que a do arco posterior e que a cortical anterior do arco posterior $(1,8 \mathrm{~mm})$ era significativamente mais espessa do que a cortical posterior $(1,0 \mathrm{~mm})$. Essas observações sugerem que se deve ter cuidado quando realizar fixação unicortical no arco posterior do atlas.

Logo abaixo e medialmente localizada à faceta articular superior, na massa lateral, existe uma pequena tuberosidade para a inserção do ligamento transverso, que 
divide o forâmen vertebral em duas partes desiguais. No nosso estudo, o diâmetro longitudinal interno foi de $31,11 \pm 2,26 \mathrm{~mm}$ na média dos observadores e de 8,86 $\pm 0,86 \mathrm{~mm}$ para a dimensão do espaço do odontóide.

\section{Conclusão}

Este estudo fornece as dimensões gerais do atlas e demonstra uma proporção constante dos arcos anterior e posterior. Os autores acreditam que essas observações possam facilitar os estudos biomecânicos, radiológicos e cirúrgicos da transição craniocervical.

\section{Referências}

1. BREATHNACH AS: Frazer's Anatomy of the Human Skeleton. Ed 6. London, J. A. Churchill, 1965, pp 29.

2. DAVEY JR, RORABECH CH, BAILEY SI: A technique of posterior cervical fusion for instability of the cervical spine. Spine 10:722-8, 1985.

3. DHALL U, CHHABRAS S, DHALL JC: Bilateral assimetry in bridges and superior articular facets of atlas vertebra. $J$ Anat Soc India 42:23-7, 1993.

4. DOHERTY BJ, HEGGENESS MHO: The quantitative anatomy of the atlas. Spine 19:2497-500, 1994.

5. DONG Y, HONG X, JIANYI L, LIN Y: Quantitative anatomy of the lateral mass of the atlas. Spine 28:860-3, 2003.

6. FOUSTAS KN, SMISSON HF, ROBINSON JSJ: C1-C2 transarticular screw fixation for atlantoaxial instability: A 6-year experience. Neurosurgery 50:672-3, 2002.

7. FRANCIS C: Dimensions of the cervical vertebrae. Anat Rec 122:603-609, 1955.

8. GRANT JBC: Grant's Atlas of Anatomy. Baltimore, Willians and Willians, 1962, pp 56.

9. GRAY H: Anatomy, Descriptive and Surgical. New York, Bounty Books, 1901, pp 36-37.

10. HISANORI M, BOYLE C, CHENG MS: Biomechanical comparison of posterior cervical fixation. Spine 26:1662-7, 2001.
11. HOHL M, BAKERH R: The atlanto-axial joint. J Bone Joint Surg 46:1739-52, 1964.

12. KANAT A, AYDIN Y: Posterior C1-C2 transarticular screw fixation for atlantoaxial arthrodesis. Neurosurgery $44: 687-9$, 1999.

13. KRAG MH: Biomechanics of the cervical spine: Internal fixation. In Frymoyer JW (ed): The Adult Spine: Principles and Practice. Ed 2. Philadelphia, Lippincot-Raven Publishers, 1997, chap 53, pp 1096-109.

14. LEVINE AM, MAZEL C, ROY-CAMILLE R: Management of fracture separations of the articular mass using posterior cervical plating. Spine 17: S447-54, 1992.

15. MADAWI AA, CASEY AT, SOLANKI GA, TUITE G, VERES R, CROCKARD HA: Radiological and anatomical evaluation of the atlantoaxial transarticular screw fixation technique. $J$ Neurosurg 86:961-8, 1997.

16. MAZARRA JT, FIELDING JW: Effects of C1- C2 rotation on canal size. Clin Orthop Rel Rs 237:115-9, 1988.

17. MONU J, BOHRER SP, HOWARD G: Some upper cervical spine norms. Spine 12:515-9, 1991.

18. NOGUEIRA-BARBOSA M: Reconstruções Multiplanares da Tomografia Computadorizada no Estudo da Artrodese Atlanto-axial Transarticular [dissertação]. Universidade de São Paulo. Ribeirão Preto, 2004.

19. PRIETO LLR, CASADO A: Assessment of the reliability of clinical findings: the intraclass correlation coeficient. Med Clin (Barc) 110:142-5, 1998.

20. RICHTER M, SCHMIDT R, CLAES L, PUHL W, WILKE $\mathrm{HJ}$ : Posterior atlantoaxial fixation: Biomechanical in vitro comparison of six different techniques. Spine 27:1724-32, 2002.

21. SHROUT PE, FLEISS JL: Intraclass correlations: uses in assessing rater reliability. Psychol Bull 86:420-8, 1979.

22. TOKUHASHIY, MATSUZAKI H, SHIRASAKI Y: C1-C2 intra articular screw fixation for atlantoaxial posterior stabilization. Spine 25:337-41, 2000.

Original recebido em julho de 2006

Aceito para publicação em março de 2007

\section{Endereço para correspondência}

Max Franco de Carvalho

Rua José Seabra Batista, 22, Condomínio Tyrol, Edificio Innsbruck 49025-000 - Aracaju, SE

E-mail: carvalhomax@hotmail.com 